\title{
The Sandwich Bone Augmentation Technique
}

\author{
Jia-Hui Fu* and Hom-Lay Wang*†
}
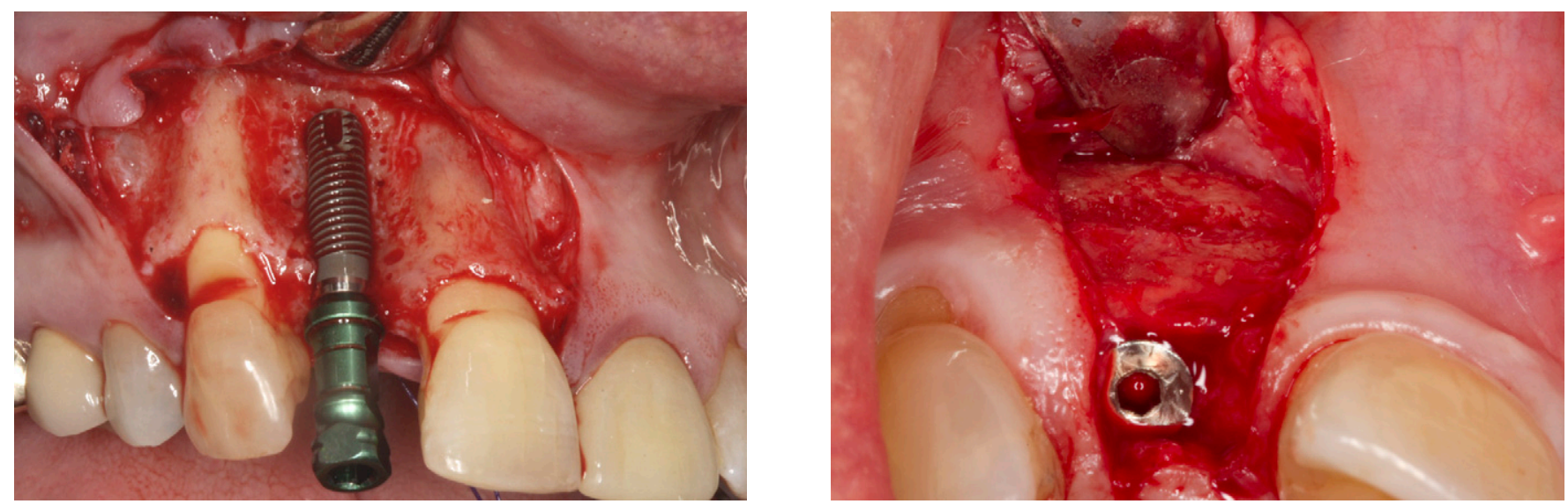

Introduction: Horizontal ridge width reduction after tooth extraction is a common clinical scenario. As such, when implant-supported restorations are planned for tooth replacement, it is a challenge to regenerate adequate bone width to house the dental implant and ensure its long-term stability. Several horizontal ridge augmentation techniques, e.g. guided bone regeneration, ridge splitting, and block grafts, have been tested and proven to predictably augment bone width. Although these techniques are successful, treatment time is significantly increased and patients need to endure additional surgical procedures. Therefore, this case report aims at illustrating the ease and success of the sandwich bone augmentation technique when performed with dental implant placement.

Case Presentation: A clinical case with a horizontally deficient ridge was selected. A buccal dehiscence was observed after placement of the dental implant in a prosthetically driven position. Autogenous bone graft harvested from the osteotomy site was placed on the exposed implant surface. Mineralized cancellous bone allograft was layered on, followed by a layer of mineralized cortical bone allograft. A collagen membrane was trimmed and used to contain the bone grafts as well as to exclude unwanted cells, such as epithelial cells and connective tissue fibroblasts. Tension-free primary closure was subsequently obtained. Six months later, mature regenerated bone was found on the buccal surface of the implant at surgical reentry.

Conclusion: Simultaneous implant placement with the sandwich bone augmentation technique predictably regenerated bone on implant buccal dehiscence defects. Clin Adv Periodontics 2012;2:172-177.

Key Words: Alveolar ridge augmentation; bone grafting; dental implants.

\section{Background}

For a dental implant to function optimally, it must be placed in a biologically acceptable and restoratively driven position. Hence, the three-dimensional positioning of the dental implant is crucial to a successful treatment outcome. However, with the loss of teeth, the alveolar bone remodels and decreases in dimensions at varying rates and degrees. ${ }^{1}$ Therefore, the clinician is often challenged with a situation in which the remaining bone support is insufficient to place

\footnotetext{
* Graduate Periodontics, Department of Periodontics and Oral Medicine, School of Dentistry, University of Michigan, Ann Arbor, MI.

† College of Dentistry, King Saud University, Riyadh, Saudi Arabia.
}

Submitted July 30, 2011; accepted for publication September 23, 2011 doi: 10.1902/cap.2012.110075 the dental implant in the ideal position. In addition, the softtissue contour is often deficient in providing an esthetically pleasing treatment outcome. ${ }^{1}$

Research showed that, during the 6-month to 2 -year postextraction period, there was a sharp reduction in the residual ridge volume, ${ }^{2}$ which continued to decrease at a much lower rate throughout life. In a 25-year longitudinal study, it was discovered that $25 \%$ of total bone width accompanied by $4 \mathrm{~mm}$ of ridge height was lost in the first year after extraction. ${ }^{3}$ In addition, a mean ridge volume loss of $40 \%$ to $60 \%$ was expected in the first 2 to 3 years after extraction. ${ }^{1}$ It was also determined that horizontal bone loss occurred before vertical bone loss $^{3}$ and to a greater extent. ${ }^{1}$

To counter the deterioration, various bone and softtissue augmentation techniques have been proposed. ${ }^{4}$ In 1988, Dahlin et al. ${ }^{5}$ were among the first to show, in a rat model, that the principle of guided tissue regeneration 
could be used to regenerate bone successfully. Guided bone regeneration (GBR) has been a well-accepted treatment modality because it has demonstrated predictable bone gain. The sandwich bone augmentation (SBA) technique is a unique form of GBR that can be used simultaneously with implant placement. ${ }^{6}$ With this technique, different bone allografts are used to simulate the composition of native bone. A layer of autograft, if readily available, is placed over the exposed implant surface, followed by a layer of cancellous bone allograft. This replicates the cancellous bone compartment in native bone. The outer layer is made up of cortical bone allograft, which thus simulates cortical bone in native bone. A barrier membrane, which functions like the periosteum, is subsequently used to contain the bone allograft and exclude non-regenerative cells that will reduce bone regeneration. With this unique blend of materials, optimal bone regeneration can be achieved when surgical principles are followed. 7 This technique significantly reduces the treatment time, eliminates a second surgical procedure, reduces cost, and also delivers positive treatment outcomes for the patients. ${ }^{8}$ This case report illustrates the ease and success of performing the SBA technique with simultaneous implant placement.

\section{Clinical Presentation and Case Management}

A healthy 51-year-old white female patient with a missing right maxillary lateral incisor (tooth \#7) was seen and treated at the Graduate Periodontics Clinic, School of Dentistry, University of Michigan. Ten years earlier, tooth \#7 was extracted without socket grafting. A written informed consent was obtained before beginning treatment. Clinically, the patient has an average smile line, moderate gingival biotype, adequate width of keratinized gingiva, and a horizontal ridge defect at site \#7 (Figs. 1a and 1b). A cone-beam computed tomography (CBCT) scan of the maxilla was taken before the surgical intervention. At site \#7, the radiographic crestal bone width, bone width $3 \mathrm{~mm}$ below the crest, and bone height was 3.12, 4 , and $16.7 \mathrm{~mm}$, respectively (Fig. 1c). The preoperative periapical radiograph depicts adequate mesio-distal space with minimal vertical ridge deficiency (Fig. 1d). The SBA technique was chosen as the technique to achieve horizontal ridge width gain simultaneous with implant placement.

Two days before surgery, the patient started a 10-day course of $500 \mathrm{mg}$ amoxicillin three times per day. Local anesthesia was obtained with buccal and palatal infiltrations using 2\% lidocaine and 1:50,000 epinephrine. Vertical incisions were made on the distal line angles of the adjacent teeth and connected to the site with intrasulcular incisions on the buccal surfaces of adjacent teeth and a mid-crestal incision at the edentulous site. Having a wide-based flap increases blood supply to the surgical site and facilitates flap advancement for primary wound closure. ${ }^{9}$ A fullthickness muco-periosteal flap was elevated and the bone width at crest and $3 \mathrm{~mm}$ apically was measured with a Boley gauge as 2.0 and $3.7 \mathrm{~mm}$, respectively (Figs. 1e and 1f). A surgical stent that indicated the ideal prosthetic position of the implant was used. The implant osteotomy was performed at $850 \mathrm{rpm}$ under copious irrigation and a $3.7-\mathrm{mm}$ diameter by $13-\mathrm{mm}$ length roughened surface implant ${ }^{\ddagger}$ was placed with $30 \mathrm{Ncm}$ at the level of the bone crest in a prosthetically driven three-dimensional position achieving primary stability. Autogenous bone graft was harvested from the implant drills after each osteotomy and stored in saline. The entire buccal surface of the implant was exposed (Figs. $1 \mathrm{~g}$ and $1 \mathrm{~h}$ ). The cover screw was secured, and intramarrow bone penetrations were performed adjacent to the implant to promote the regional acceleratory phenomenon. ${ }^{10}$ Intramarrow bone penetrations were performed using a quarter round diamond bur positioned perpendicular to the bone surface and drilled past the cortical plate and slightly into the cancellous bone. Autogenous bone graft harvested from the osteotomy site was subsequently placed on the exposed implant surface. However, it has limited availability, and a second surgical site is often needed. ${ }^{11}$ Therefore, human allograft is the next best choice. ${ }^{8}$ Mineralized cancellous bone allograft ${ }^{\S}$ was layered on the surface of the autogenous graft to the level of the adjacent bone. Furthermore, to compensate for potential graft resorption, a 2- to 3-mm thick layer of mineralized cortical bone allograft" was added on top of the cancellous allograft (Figs. 1i and 1j). Layering the mineralized cancellous and cortical bone allografts takes advantage of the creeping and reverse creeping substitution healing processes and also mimics the macrostructure of native bone. ${ }^{11}$ Creeping substitution occurs in the healing of cancellous bone grafts. In this process, new bone apposition, involving an influx of angiogenesis and osteoblastic activity, occurs and is followed by bone resorption and remodeling. Cortical bone grafts, conversely, undergo reverse creeping substitution, in which the grafts go through an initial bone resorption phase, followed by bone formation. Therefore, there will be a period of time in which cortical grafts remain as a mixture of necrotic and vital bone, at which time they have the weakest strength. A mineralized bone allograft is chosen because it will maintain the space for bone regeneration for a longer period of time compared to demineralized bone allograft. ${ }^{12}$ A collagen membrane was trimmed and used to contain the bone grafts and also exclude the nonregenerative cells, such as epithelial cells from colonizing the site (Fig. 1k). This will enable osteoprogenitor cells to colonize the site and use the allografts as a scaffold to regenerate new vital bone. Collagen membranes are preferred because they are absorbable, biocompatible, and enhance hemostasis and chemotaxis of fibroblasts. In addition, a second surgical procedure to remove the membrane is eliminated, increasing patient satisfaction while reducing site morbidity, attachment loss, and treatment time. ${ }^{11}$ Periosteal flap release was performed to ensure that the flaps were sutured with tension-free primary closure (Fig. 11). This technique provides the regenerative site with a protected, stable, and undisturbed healing

\footnotetext{
‡ Tapered Screw-Vent Implant, Zimmer Dental, Carlsbad, CA.

$\S$ Puros, Zimmer Dental.

" Puros, Zimmer Dental.

ฯ Pericardium Membrane, Zimmer Dental.
} 

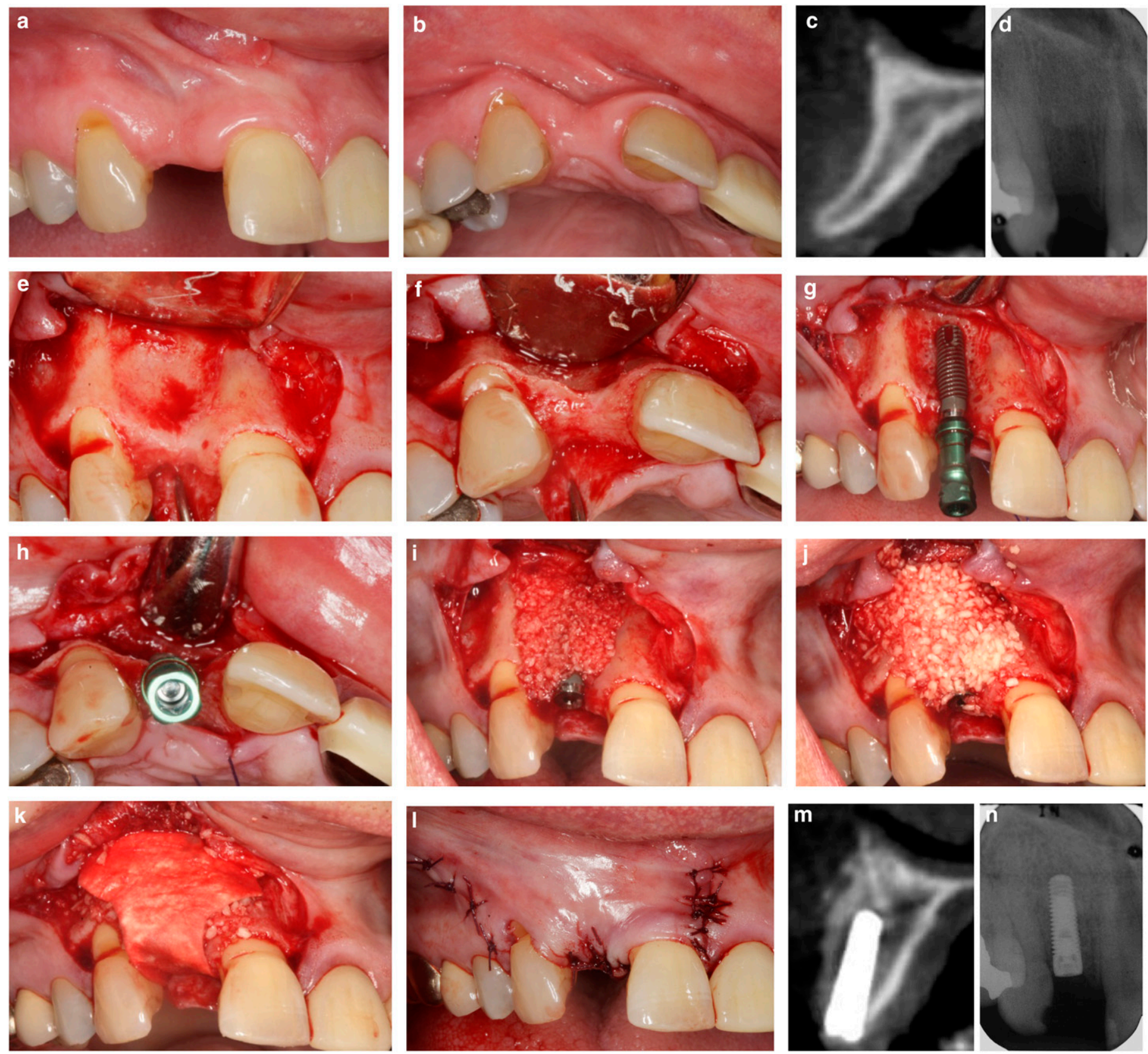

FIGURE 1a Preoperative buccal view of the edentulous site at tooth \#7. 1b Preoperative occlusal view of the edentulous site at tooth \#7. 1c Midsagittal CBCT view of ridge at tooth \#7. 1d Preoperative periapical radiograph of site \#7. 1e Buccal view of surgical site. 1f Occlusal view of surgical site. 1g Extensive buccal dehiscence on implant surface. $1 \mathrm{~h}$ Implant was placed in prosthetically driven position. 1i Mineralized cancellous bone allograft placed on buccal surface of the implant. 1j Mineralized cortical bone allograft placed on buccal surface of the implant. 1k Absorbable barrier membrane positioned. 1I Primary wound closure achieved. 1m Postoperative CBCT scan. 1n Periapical radiograph of implant placement

phase. ${ }^{7}$ A postoperative CBCT scan was taken, and 1.5 $\mathrm{mm}$ bone allograft was found buccal to the implant surface at the implant neck and $3 \mathrm{~mm}$ apically (Fig. $1 \mathrm{~m}$ ). A postoperative periapical radiograph demonstrated the mesiodistal and vertical positions of the implant (Fig. 1n). The site was left to heal for the next 6 months.

\section{Clinical Outcomes}

Reentry surgery was performed 6 months after implant placement (Figs. 2a and 2b). A CBCT scan was obtained, and radiographic bone width buccal to the implant surface at the implant neck, $3 \mathrm{~mm}$ apically, and $6 \mathrm{~mm}$ apically was
$0,0.85$, and $2 \mathrm{~mm}$, respectively (Fig. 2c). A full-thickness muco-periosteal flap, with two vertical incisions, was reflected at the implant site. Mature bone was observed on the entire buccal surface of the implant (Fig. 2d). A bone biopsy was performed, and histologic analysis showed mature bone formed around particles of bone allograft (Fig. 2e). A healing abutment was secured and the flaps sutured (Fig. 2f). The implant was restored with a porcelain fused to metal cement-retained crown 1 month after (Figs. $2 \mathrm{~g}$ and $2 \mathrm{~h}$ ) and followed up for 1 year (Figs. $2 \mathrm{i}$ and $2 \mathrm{j}$ ). A periapical radiograph taken 1 year after implant placement showed normal bone levels to the first implant thread (Fig. 2k). 

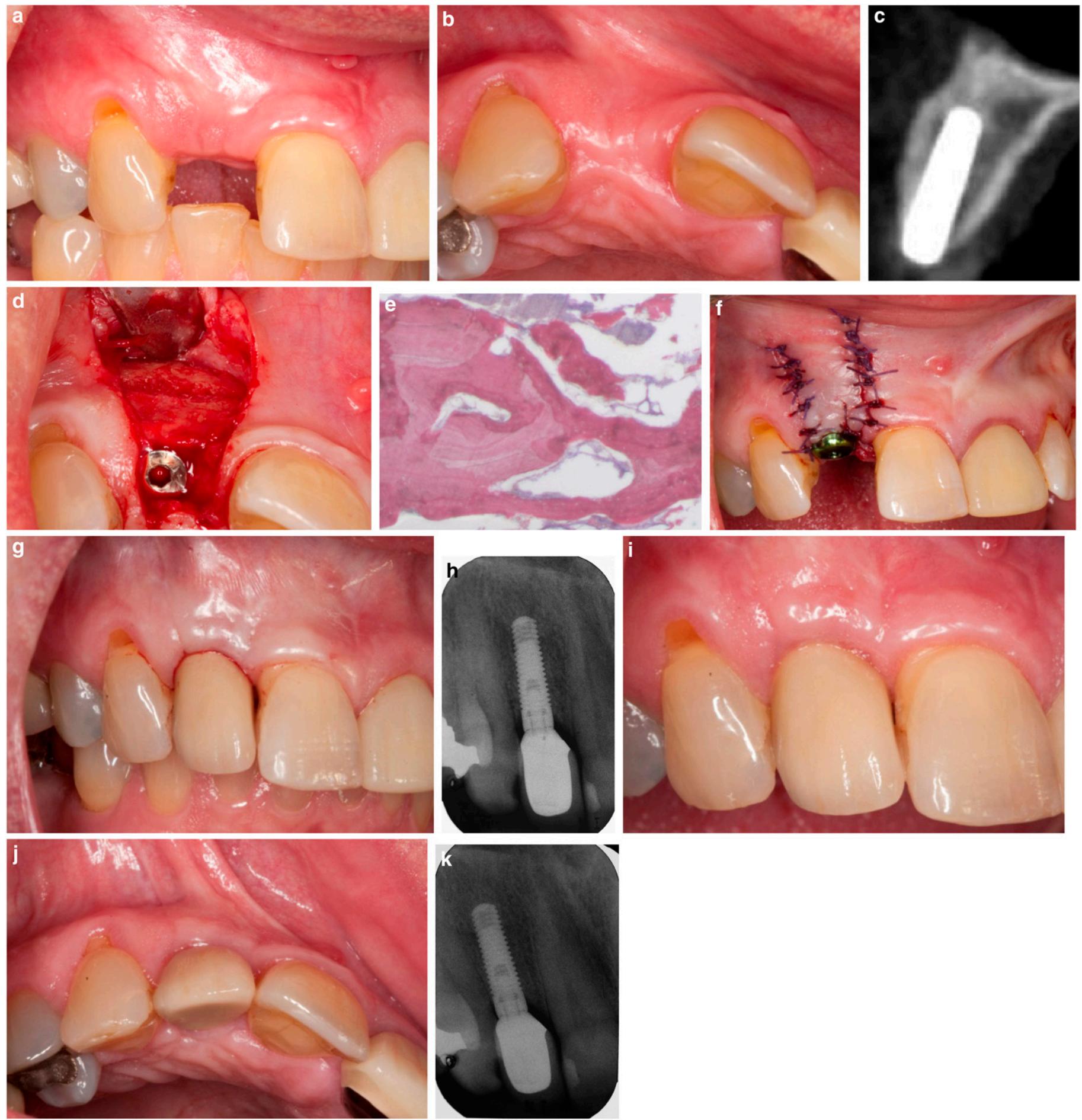

FIGURE 2a Preoperative buccal view. 2b Preoperative occlusal view. 2c Midsagittal CBCT view 6 months after implant placement. 2d Mature bone observed on the entire buccal implant surface. 2e Histologic image of mature bone formation around particles of bone allograft (Hematoxylin and eosin stain. Original magnification $\mathrm{x} 4)$. $2 \mathrm{f}$ Healing abutment secured with flaps sutured. $2 \mathrm{~g}$ Crown was delivered. $2 \mathrm{~h}$ Periapical radiograph of crown at delivery. 2i Buccal view of crown at 1 year after implant placement. 2j Occlusal view of crown at 1 year after implant placement. 2k Periapical radiograph of crown at 1 year after implant placement.

\section{Discussion}

The "PASS" principle (which stands for achieving primary wound closure, promoting angiogenesis, maintaining space for regeneration and obtaining primary implant, and blood clot stability) is aimed at promoting bone regeneration. ${ }^{7}$ The SBA technique is designed to follow this principle in an attempt to achieve optimal bone regeneration. Performing periosteal flap release increases the mobility of the flap and reduces flap tension, thereby enabling the blood clot to be undisturbed and the surgical site to remain closed and protected during healing. Studies have showed a reduction in bone volume regenerated when a surgical site is exposed, possibly as a result of bacterial colonization, reduced blood supply to the site, and contamination from exogenous 
agents. ${ }^{8}$ Intramarrow bone penetrations were performed around the implant to promote an influx of growth factors and osteoprogenitor cells from the bone marrow to the implant and bone allograft site. ${ }^{13}$ The major limitation of this technique is the technical difficulty associated with achieving primary implant stability in a horizontally challenged ridge. This is particularly important because any micromovement may result in fibrous encapsulation of the implant and its eventual removal. ${ }^{14}$

Preliminary studies have shown that the SBA technique is predictable and reliable in achieving bone regeneration with implant placement.6,8 This technique provides patients with a shortened treatment time, predictable restoration, and reduced morbidity, discomfort, and cost. It also has a relatively short learning curve that clinicians can master and appreciate. However, there is a certain degree of technical difficulty associated with stabilizing the dental implant in a narrow residual ridge. Therefore, clinicians have to be adequately trained before using this technique routinely.

There are several limitations associated with this technique. They include membrane exposure, inability of the absorbable membrane to maintain space needed for regeneration, and achieving primary implant stability. An experimental study has demonstrated that the most ideal regeneration occurred when membranes remained unexposed for a minimum of 6 to 8 months. ${ }^{15}$ Therefore, ensuring adequate flap advancement so that stable tension-free wound closure is achieved is one method to avoid membrane exposure. Bone grafts are also used in conjunction with absorbable barrier membranes to help in space maintenance. Hence, in this technique, mineralized allografts were used. Furthermore, an appreciation for anatomic structures and bone density is important when placing dental implants so that mechanical interlocking of the fixture to bone is attained.

\section{Summary}

Why is this case new information? management of this case?

What are the primary limitations to success in this case?
- This case report illustrates the ability of the SBA technique with simultaneous implant placement to predictably regenerate bone on extensive buccal dehiscence defects on implants.

- The key to successful management of this case is following the PASS principle.

- The primary limitations are membrane exposure, collapse of membrane, and loss of primary implant stability.

\section{Acknowledgments}

Drs. Fu and Wang have received financial support for research from Zimmer Dental (Carlsbad, California). Dr. Wang has also received lecture fees from Zimmer Dental.
CORRESPONDENCE:

Dr. Hom-Lay Wang, Department of Periodontics and Oral Medicine, University of Michigan School of Dentistry, 1011 N. University Ave., Ann Arbor, MI 48109-1078. E-mail: homlay@umich.edu. 


\section{References}

1. Schropp L, Wenzel A, Kostopoulos L, Karring T. Bone healing and soft tissue contour changes following single-tooth extraction: A clinical and radiographic 12-month prospective study. Int J Periodontics Restorative Dent 2003;23:313-323.

2. Tallgren A. Positional changes of complete dentures. A 7-year longitudinal study. Acta Odontol Scand 1969;27:539-561.

3. Tallgren A. The continuing reduction of the residual alveolar ridges in complete denture wearers: A mixed-longitudinal study covering 25 years. J Prosthet Dent 1972;27:120-132.

4. Prato GP, Cairo F, Tinti C, Cortellini P, Muzzi L, Mancini EA. Prevention of alveolar ridge deformities and reconstruction of lost anatomy: A review of surgical approaches. Int J Periodontics Restorative Dent 2004;24:434-445.

5. Dahlin C, Linde A, Gottlow J, Nyman S. Healing of bone defects by guided tissue regeneration. Plast Reconstr Surg 1988;81:672-676.

6. Wang HL, Misch C, Neiva RF. "Sandwich" bone augmentation technique: Rationale and report of pilot cases. Int J Periodontics Restorative Dent 2004;24:232-245.

7. Wang HL, Boyapati L. "PASS" principles for predictable bone regeneration. Implant Dent 2006;15:8-17.

indicates key references.
8. Park SH, Lee KW, Oh TJ, Misch CE, Shotwell J, Wang HL. Effect of absorbable membranes on sandwich bone augmentation. Clin Oral Implants Res 2008;19:32-41.

9. Park SH, Wang HL. Mucogingival pouch flap for sandwich bone augmentation: Technique and rationale. Implant Dent 2005;14:349-354.

10. Frost HM. The regional acceleratory phenomenon: A review. Henry Ford Hosp Med J 1983;31:3-9.

11. Lee A, Brown D, Wang HL. Sandwich bone augmentation for predictable horizontal bone augmentation. Implant Dent 2009;18:282-290.

12. Wang HL, Cooke J. Periodontal regeneration techniques for treatment of periodontal diseases. Dent Clin North Am 2005;49:637-659, vii.

13. Greenstein G, Greenstein B, Cavallaro J, Tarnow D. The role of bone decortication in enhancing the results of guided bone regeneration: A literature review. J Periodontol 2009;80:175-189.

14. Brunski JB, Moccia AF Jr, Pollack SR, Korostoff E, Trachtenberg DI. The influence of functional use of endosseous dental implants on the tissue-implant interface. II. Clinical aspects. J Dent Res 1979;58:19701980.

15. Lekholm U, Becker W, Dahlin C, Becker B, Donath K, Morrison E. The role of early versus late removal of GTAM membranes on bone formation at oral implants placed into immediate extraction sockets. An experimental study in dogs. Clin Oral Implants Res 1993;4:121-129. 